\title{
ORQUIDEOFLÓRULA DE UN SECTOR DE SERRANÍA DE LA CUCHILLA, MUNICIPIO CARIPE, ESTADO MONAGAS, VENEZUELA
}

\author{
Carlos Leopardi
}

\begin{abstract}
Centro de Investigación Científica de Yucatán, Postgrado en Ciencias Biológicas, Mérida 97200, México.
Herbario Isidro Ramón Bermúdez Romero, Departamento de Biología, Universidad de Oriente, Núcleo de Sucre, Cumaná 6101 Apdo. 245, Sucre, Venezuela. leopardiverde@gmail.com
\end{abstract}

\begin{abstract}
RESUMEN. A fin de contribuir con el conocimiento de la familia Orchidaceae en el Macizo del Turimiquire, se realizaron exploraciones en un sector de la Serranía de La Cuchilla, municipio Caripe, estado Monagas $\left(10^{\circ} 07,00^{\prime} \mathrm{N} 63^{\circ} 33,45^{\prime} \mathrm{O}\right)$ entre los años 2005-2008 y durante los meses de diciembre-enero, mayo, julio-septiembre. Todos lo ejemplares colectados están depositados en IRBR con duplicados en VEN. Se identificaron 47 especies en 30 géneros. Los géneros más importantes son: Epidendrum ( 8 spp.), Pleurothallis s.l. (3 spp.) y Prosthechea (3 spp.), y las especies más frecuentes fueron: Epidendrum subpurum, Acianthera lanceana y Scaphyglottis fusiformis. Así mismo, se señalan un nuevo reporte para la porción oriental de la Cordillera de la Costa: Acianthera miqueliana. Finalmente, el $20 \%$ de las especies mencionadas está señalado en el Libro Rojo de la Flora Venezolana: una en peligro crítico (Cattleya gaskelliana), tres especies vulnerables, una en peligro tres en menor riesgo/casi amenazadas y una (Prosthechea venezuelana) como insuficientemente conocida. Se presentan fotografías de algunas de las especies encontradas y una clave para su identificación.
\end{abstract}

AвSTRAct. To contribute with the study of the Orchidaceae on El Macizo Montañoso del Turimiquire, field exploration was done in a sector of the Serranía de La Cuchilla, Caripe municipality, Monagas state $\left(10^{\circ} 07,00^{\prime} \mathrm{N} 63^{\circ} 33,45^{\prime} \mathrm{O}\right)$, during the years $2005-2008$, in the months of Decembe-January, May and JulySeptember. Collected specimens were housed in the Herbarium IRBR with duplicates at VEN. As result, 47 species in 30 genera were identified. The most importantr genera are Epidendrum (8 spp.), Pleurothallis s.l. (3 spp.) and Prosthechea (3 spp.); the most frequent species are: Epidendrum subpurum, Acianthera lanceana y Scaphyglottis fusiformis. Additionally, a new record to the east portion of Cordillera de la Costa is reported: Acianthera miqueliana. Finally, $20 \%$ of species are mentioned in the Red Book of Venezuelan Flora: one in critic danger (Cattleya gaskelliana), three as vulnerable, one in danger, three as minor risk, and one (Prosthechea venezuelana) as insufficiently known. An identification key to the species and pictures of some of the taxa are presented.

Palabras ClaVes / Key words: Orchidaceae, Florística, Floristic, Macizo del Turimiquire, Monagas, Venezuela

La familia Orchidaceae es uno de los grupos de Magnoliophyta más ampliamente distribuido a nivel mundial (Dressler 1981); así, como uno de los más diversos, con más de 24000 especies (Chase et al. 2003), la mayoría de éstas en el continente americano, especialmente en la cordillera andina (Dressler, 1981; Carnevali et al. 2007). En Venezuela, esta familia alcanza aproximadamente 1612 especies en 222 géneros (Carnevali et al., 2007), siendo las áreas con el mayor número de especies la Cordillera de Mérida y la Sierra de Perijá, que en conjunto poseen una orquideoflora compuesta por más de 818 especies, seguidas de la Guayana venezolana con más de 700 especies (Carnevali et al. 2003, 2007).
En el nororiente de Venezuela se han realizado algunos trabajos donde se han producido listas de especies. Entre ellos destacan las exploraciones de Steyermark al cerro Turimiquire y la región oriental adyacente (Schweinfurt 1957; Steyermark 1966), las referencias presentadas por Dunsterville y Garay (1959, 1961, 1965, 1966, 1972, 1976) a lo largo de su obra a colecciones realizadas en Caripe y áreas adyacentes, los reportes de Foldats $(1969,1970 \mathrm{a}, 1970 \mathrm{~b}, 1970 \mathrm{c}, 1970 \mathrm{~d})$ de las colecciones realizadas por varios exploradores en el Macizo del Turimiquire, el listado de especies para el Parque Nacional El Guácharo presentado por Lárez (2003), entre otros. Sin embargo, estas exploraciones usualmente involucran todos los componentes de la 
flora, por lo que hasta ahora, en el nororiente y muy especialmente en el Macizo del Turimiquire no es posible establecer cuál es el nivel real de conocimiento de la familia Orchidaceae. En este sentido, se presenta un listado, tentativo, de las especies de orquídeas presentes en un sector de la Serranía de la Cuchilla, sur del Macizo Montañoso del Turimiquire, en un área cercana al Parque Nacional El Guácharo.

\section{Materiales y métodos}

Área de estudio

Las muestras se colectaron en La Hacienda La Cuchilla (Fig. 1A), que se encuentra en la Serranía de la Cuchilla (sur del Macizo del Turimiquire), al sur del municipio Caripe, en los límites con el municipio Piar $\left(10^{\circ} 07^{\prime} 00^{\prime} \mathrm{N}\right.$ 6 633' $45^{\prime}$ 'O), estado Monagas, Venezuela. Altitudinalmente, se ubica entre los 900$1200 \mathrm{~m} \mathrm{snm}$. Desde el punto de vista climático, tiene temperaturas que oscilan entre los 12 y los $24^{\circ} \mathrm{C}$. Las precipitaciones alcanzan $1124 \mathrm{~mm}$ anuales, presentando un claro patrón de biestacional, con un período de sequía que va desde enero hasta mayo y uno lluvioso que va desde junio hasta diciembre [datos climáticos desde http://venciclopedia.com/index.php?title=Municipio_ Caripe_(Monagas)].

\section{Métodos}

Entre los años 2005-2008, durante los meses de diciembre-enero, mayo, julio-septiembre, se realizaron exploraciones dentro de los límites de los cafetales, considerándose especies terrestres, epífitas o litófitas. Las epífitas, generalmente, se colectaron de las ramas de árboles caídos. Todas las especies colectadas fueron mantenidas en cultivo, teniendo especial cuidado con aquellas que al momento de colección no se encontraban en fenofase reproductiva. Las muestras para herbario se tomaron generalmente en dos partes, una para la exsiccata, que se procesó según las técnicas tradicionales para la preparación de muestras de herbario (Lindorf et al., 1999) y otra constituida sólo de flores, que fueron fijadas en FAA, de las que se utilizó parte durante la identificación. El material fue identificado con la ayuda de las obras de Foldats (1969, 1970a, 1970b, 1970c, 1970d), Steyermark y Huber (1978), Luer (1986a, 1986b), Romero y Carnevali (2000) y Carnevali et al. (2003). De forma general se sigue el sistema propuesto por Pridgeon et al. (2003, 2005, 2009). Las fotografías fueron tomadas por el autor en condiciones de campo o cultivo. Las muestras colectadas fueron depositadas en el IRBR con duplicados en VEN [testigos de herbario: Leopardi s.n (todas las muestras en líquido año 20052006 y parte de las de 2007-2008), Leopardi et al. 150207, 300-313].

\section{Resultados y discusión}

Se identificaron 47 especies de Orchidaceae en 30 géneros; siendo los mejor representados Epidendrum L. (8 spp.), Pleurothallis R. Br. s.l. (3 spp.) y Prosthechea Knowles \& Westc. (3 spp.) (Tabla 1). La mayor parte de las especies reportadas son epífitas. El forofito (hospedero) más frecuente son los Guamos (Inga spp.); sin embargo, los forofitos con mayor diversidad usualmente son los árboles de gran tamaño, como el Bucare (Erythrina sp.), Angelino (Homalium sp.), entre otros.

Las especies más frecuentes son Epidendrum subpurum Rchb. f., Acianthera lanceana (Lodd.) Pridgeon \& M.W. Chase y Scaphyglottis fusiformis (Griseb.) Schult. Otras como Epidendrum secundum Jacq. o Cyrtopodium aff. willmorei Knowles \& Westc., se encuentran sólo en las porciones sabanizadas de la serranía y algunas especies como Govenia utriculata (Sw.) Lindl. prefieren lugares sombreados con alta cantidad de materia orgánica.

La alta proporción de especies epífitas con respecto a las terrestres/litófitas ya ha sido señalada por Gentry y Dodson (1987) al indicar que más del 70\% de las especies conocidas de orquídeas prefieren esa forma de vida; aunado a esto, las prácticas culturales para la manutención de los cafetales tienden a eliminar las plantas que crecen en el suelo, encontrándose éstas sólo en lugares protegidos, entre raíces tabulares de algunos árboles, muy cerca de troncos de árboles en descomposición (usualmente sobre o tendiendo a estar debajo de ellos) o en lugares no cultivados, a veces cerca de los caminos, como es el caso de Epidendrum secundum.

Por otro lado, la alta frecuencia de algunas plantas como Epidendrum subpurum o Acianthera lanceana podría asociarse al carácter secundario de la vegetación y a las características microclimáticas asociadas a los distintos hábitats, ya que géneros como Dichaea Lindl. tienen tendencia a crecer en lugares sombríos y húmedos, que no siempre están garantizados en los cafetales, por lo que usualmente se les puede encontrar en la zona límite 
Tabla 1. Orchidaceae en la Hacienda La Cuchilla, municipio Caripe, estado Monagas, Venezuela.

\section{Nombre Científico}

Acianthera lanceana (Lodd. ex Lindl.) Pridgeon \& M.W.Chase

A. miqueliana ${ }^{+}$(Lodd. ex Lindl.) Pridgeon \& M.W.Chase

A. rubroviridis (Carnevali \& G.A. Romero) Carnevali \& G.A. Romero

Anathallis sclerophylla (Lindl.) Pridgeon \& M.W. Chase

Brassia wageneri Rchb.f.

Camaridium micranthum M. A. Blanco.

Camaridium ochroleucum Lindl.

Campylocentrum micranthum (Lindl.) Rolfe

Cattleya gaskelliana Rchb.f.

Cyrtopodium aff. willmorei Knowles \& Westc.

Dimerandra emarginata (G.Mey.) Hoehne

Elleanthus graminifolius (Barb.Rodr.) Løjtnant

Encyclia cordigera (Kunth) Dressler

Epidendrum anceps Jacq.

E. ciliare L.

E. ferrugineum Ruiz \& Pav.

E. ramosum Jacq.

E. rigidum Jacq.

E. secundum vel sp. aff. Jacq.

Epidendrum aff. siphonosepalum Garay \& Dunst.

E. subpurum Rchb.f.

Gomesa bicolor (Lindl.) M.W.Chase \& N.H.Williams

Gongora maculata Lindl.

Govenia utriculata vel sp. aff. (Sw.) Lindl.

Isochilus linearis (Jacq.) R.Br.

Jacquiniella globosa (Jacq.) Schltr.

J. teretifolia (Sw.) Britton \& P. Wilson

Leochilus labiatus (Sw.) Kuntze

Lockhartia sp.

Maxillaria porrecta Lindl.

Maxillariella caespitifica (Rchb.f.) M. A. Blanco \& Carnevali

Mormodes atropurpurea Lindl.

Mormodes sp.

Oeceoclades maculata (Lindl.) Lindl.

O. obryzatum Rchb.f. \& Warsz.

Pleurothallis discoidea Lindl.

P. pruinosa Lindl.

P. revoluta (Ruiz \& Pav.) Garay

Polystachya foliosa (Hook.) Rchb.f.

Prosthechea chacaoensis (Rchb.f.) W.E.Higgins

P. livida (Lindl.) W.E. Higgins

$P$. venezuelana (Schltr.) W.E.Higgins

Rodriguezia lanceolata Ruiz \& Pav.

Scaphyglottis fusiformis (Griseb.) R.E.Schult.

Stanhopea wardii Lodd. ex Lindl.

Stelis cucullata Ames

S. pygmaea Cogn.

\begin{tabular}{|c|c|}
\hline$S$ & EC \\
\hline$E$ & $\mathrm{MR} / \mathrm{ca}$ \\
\hline$E$ & - \\
\hline$E$ & - \\
\hline$E$ & - \\
\hline$E$ & - \\
\hline$E$ & - \\
\hline$E$ & - \\
\hline$E$ & - \\
\hline$E$ & $\mathrm{CR}$ \\
\hline $\mathrm{T}$ & - \\
\hline$E$ & - \\
\hline$E$ & - \\
\hline$E$ & VU \\
\hline$E$ & - \\
\hline$E$ & - \\
\hline$E$ & - \\
\hline$E$ & - \\
\hline$E / L$ & - \\
\hline $\mathrm{E} / \mathrm{T}$ & - \\
\hline$E$ & - \\
\hline$E$ & - \\
\hline$E$ & VU \\
\hline$E$ & - \\
\hline $\mathrm{T}$ & - \\
\hline$E$ & - \\
\hline$E$ & - \\
\hline$E$ & - \\
\hline$E$ & - \\
\hline$E$ & - \\
\hline$E$ & - \\
\hline$E$ & - \\
\hline ED & EP \\
\hline ED & - \\
\hline $\mathrm{T}$ & - \\
\hline$E$ & - \\
\hline$E$ & - \\
\hline$E$ & - \\
\hline$E$ & - \\
\hline$E$ & - \\
\hline$E$ & $\mathrm{MR} / \mathrm{ca}$ \\
\hline$E$ & - \\
\hline$E$ & IC \\
\hline$E$ & $\mathrm{MR} / \mathrm{ca}$ \\
\hline$E$ & - \\
\hline$E$ & VU \\
\hline$E$ & - \\
\hline$E$ & - \\
\hline
\end{tabular}

$+=$ nuevo registro para el Macizo del Turimiquire

$\mathrm{S}=$ sustrato; $\mathrm{E}=$ epífita; $\mathrm{T}=$ terrestre; $\mathrm{L}=$ litófita; $\mathrm{ED}=$ epífita en troncos en descomposición.

$\mathrm{EC}=$ Sistema de categorías de la Lista Roja, según Llamozas et al. (2003); $\mathrm{MR} / \mathrm{ca}=$ menor riesgo/casi amenazada; CR = peligro crítico; EP = En Peligro; VU = vulnerable; IC = insuficientemente conocida; - = no hay datos disponibles. 
entre el cafetal y el área boscosa no cultivada.

Es notable que, a pesar de que el área estudiada involucra menos de 100 ha, cuando se compara con otros trabajos realizados en el municipio Caripe o áreas cercanas, la diferencia en el número de especies reportadas es considerable; así, Steyermark en su exploración al cerro Turimiquire y región oriental adyacente reporta 61 especies repartidas entre los estados Anzoátegui (34 spp.), Monagas (24 spp.) y Sucre (11 spp.), siendo las de Sucre colectadas casi todas (10 spp.) en el Cerro Turimiquire y las de Monagas en su mayoría (21 spp.) en el municipio Caripe y una de ellas, Sacoila lanceolata (Aubl.) Garay [como Spiranthes orchioides (Sw.) A. Rich. en Schweinfurth, Fieldiana, Bot. 28 (3-4): 862. 1957], en las sabanas de la Serranía de La Cuchilla (Schweinfurth 1957).

En exploraciones posteriores al Cerro Turimiquire, Steyermark refiere una lista de 17 especies (Steyermark 1966), mientras que Figueroa (1992) menciona 8 especies para la flórula del Cerro de la Cueva del Guácharo (Parque Nacional El Guácharo); posteriormente, Lárez (2003) señala 11 spp. para el Parque Nacional el Guácharo; en general, las especies mencionadas por Lárez (2003) y Figueroa (1992) coinciden con las reportadas por Steyermark (1966). De los trabajos publicados, el que tiene el número más cercano a lo reportado en este trabajo es el Foldats (1969, 1970a, 1970b, 1970c, 1970d) que recopilando todas las colecciones realizadas hasta los años 60 menciona $c a$. 37 especies para el municipio Caripe. Lo antes señalado podría atribuirse al esfuerzo de colección de los distintos autores; al respecto, Carnevali et al. (2007) comentan que independientemente de lo bien que haya sido trabajada un área por botánicos convencionales, dado lo críptico de la mayoría de las especies de orquídeas, cuando un botánico especializado en Orchidaceae explora sistemáticamente esa misma área, usualmente aparecen nuevos registros para la flora local, esto está en concordancia con Lárez (2005), que señala que el hábito epífito de estas plantas dificulta su colección.

$\mathrm{Al}$ revisar la distribución de las plantas, una resultó nueva para la región nororiental del país: Acianthera miqueliana (H. Focke) Pridgeon \& M.W.Chase que según Carnevali et al. (2003) ha sido colectada fuera de la Guayana venezolana en la porción central y occidental de la Cordillera de la Costa; sin embargo, existe la posibilidad de que esta entidad pueda ser una especie muy afin, Acianthera angustifolia (Lindl.) Luer, que también ha sido colectada en la porción central de la cordillera de la costa (G. Carnevali, com. pers. 2009).

Cuando se revisa la lista de familias con el mayor número de especies para el estado Monagas, publicada por Lárez (2005), se aprecia la ausencia de la familia Orchidaceae, que considerando sólo las especies señaladas en este trabajo, pasaría a estar el en noveno lugar de importancia para el estado y al menos, en el tercer lugar de importancia para la Cordillera de la Costa, esto considerando la información proporcionada por Lárez (2005) sobre los principales ecosistemas del estado Monagas (Llanos, Piedemonte y Cordillera de la Costa).

Finalmente, al revisar el estado de conservación de las especies señalas en el Libro Rojo de la Flora Venezolana (Llamozas et al. 2003), se encuentra que el $20 \%$ está bajo alguna categoría (Tabla 1). Resulta inquietante que una está en peligro crítico: Cattleya gaskelliana Rchb. f., endémica del Macizo del Turimiquire; aparte, 3 están catalogadas como vulnerables, 1 está catalogada En Peligro (Mormodes atropurpurea Lindl.), 3 como menor riesgo/casi amenazadas y una [Prosthechea venezuelana (Schltr.) W.E. Higgins] como insuficientemente conocida.

\section{Clave Para la identificación DE LAS especies}

1a. Hojas teretes, triquetras (de contorno circular o triangular) o lateralmente aplanadas ............................. 2

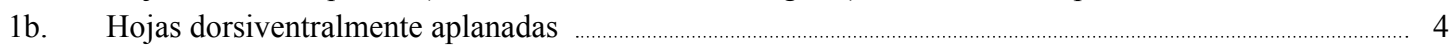

2a. Hojas lateralmente aplanadas, imbricadas _........................................................................... Lockhartia sp.

2b. Hojas teretes o algo triquetras, no imbricadas

3a. Plantas de menos de $7 \mathrm{~cm}$ de alto. Inflorescencia sin brácteas foliáceas en su ápice

Jacquiniella globosa

3b. Plantas de más $10 \mathrm{~cm}$ de alto. Inflorescencia con 1--2 brácteas foliáceas en su ápice

Jacquiniella teretifolia 
4a. Pseudobulbos ausentes

4b. Pseudobulbos presentes...

5a. Crecimiento monopodial

5b. Crecimiento simpodial

6a. Flores generalmente solitarias; labelo sin espolón

Epidendrum aff. siphonosepalum

6b. Flores en racimos; labelo espolonado Campylocentrum micranthum

7a. Ovario no articulado con el pedicelo 8

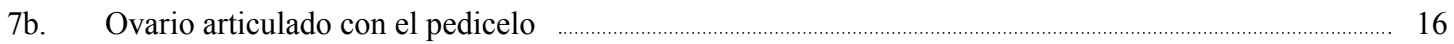

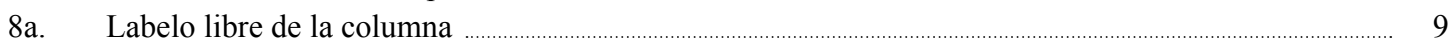

8b. Labelo unido a la columna …….......................................................................................................................... 11

9a. Flores agrupadas en inflorescencia espiciforme. Brácteas conspicuas, cubriendo a las flores. Flores blancas Elleanthus graminifolius

9b. Flores solitarias, sucesivas, raramente 2. Brácteas inconspicuas. Flores rosadas 10

10a. Tallos secundarios ligeramente engrosados. Hojas de más de $0.5 \mathrm{~cm}$ de ancho. Flor de más de $1 \mathrm{~cm}$ de diámetro; labelo basalmente con un callo compuesto por una serie de lamelas transversales

Dimerandra emarginata

10b. Tallos secundarios no engrosados. Hojas de hasta $0.3 \mathrm{~cm}$ de ancho. Flor de menos de $0.6 \mathrm{~cm}$ de diámetro; labelo ecalloso

Isochilus linearis

11a. Labelo simple

11b. Labelo 3-lobulado

12a. Ovario con una vesícula alargada en su ápice

Epidendrum ramosum

12b. Ovario sin una vesícula alargada en su ápice

Epidendrum rigidum

13a. Inflorescencia péndula. Lóbulo medio del labelo agudo Epidendrum ferrugineum

13b. Inflorescencia erecta. Lóbulo medio del labelo truncado-retuso hasta emarginado

14a. Inflorescencia racemosa. Labelo con márgenes irregularmente dentado-laciniados

Epidendrum secundum vel aff.

14b. Inflorescencia racemosa o paniculada. Labelo con márgenes enteros

15a. Hojas oblongas. Inflorescencia subcapitada

Epidendrum anceps

15b. Hojas lineares. Inflorescencia no subcapitada Epidendrum subpurum

16a. Sépalos unidos entre sí, flores de contorno triangular

16b. Sépalos libres o unidos, flores nunca de contorno triangular

17a. Inflorescencia con menos de 4 flores, laxas. Labelo apiculado Stelis pygmaea

17b. Inflorescencia con más de 6 flores. Labelo obtuso Stelis cucullata

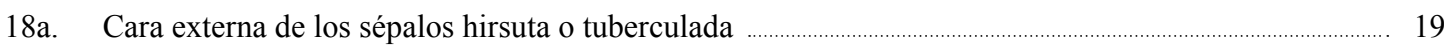

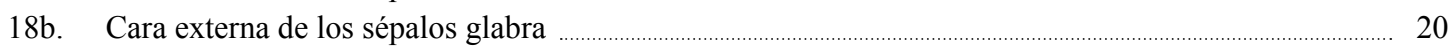

19a. Inflorescencia racemosa con más de 4 flores. Pétalos lanceolados con los márgenes fimbriados. Ovario glabro Acianthera lanceana

19b. Inflorescencia fasciculada, con 1--3 flores. Pétalos flabelados de márgenes enteros. Ovario pubescente

20a. Rizoma alargado, ramicaules visiblemente separados entre sí. Hojas moradas Acianthera rubroviridis

20b. Rizoma abreviado, ramicaules muy juntos. Hojas generalmente verdes Acianthera miqueliana

21a. Flores amarillas, sucesivas o raro 2 21

21b. Flores blanquecinas y con antesis simultánea Pleurothallis discoidea

22a. Plantas con menos de $6 \mathrm{~cm}$ de alto. Inflorescencia de 2--4 flores 22

22b. Plantas con más de $7 \mathrm{~cm}$ de alto. Inflorescencia con más de 6 flores Pleurothallis pruinosa

23a. Sépalos glabros en su cara interna; pétalos linear-filiformes, de longitud similar a la de los sépalos; labelo geniculado

Pleurothallis revoluta 
23b. Sépalos pubescentes en su cara interna; pétalos elípticos, más pequeños que los sépalos; labelo no geniculado Anathallis sclerophylla

24a. Labelo unido a la columna, profundamente 3-lobulado; lóbulo medio acicular, lóbulos laterales con el margen externo irregularmente laciniado Epidendrum ciliare

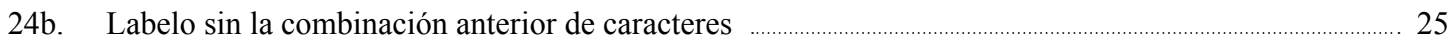

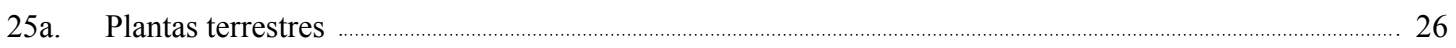

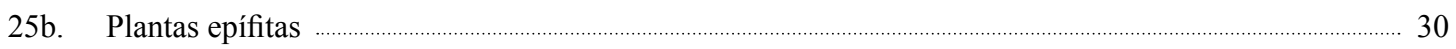

26a. Pseudobulbos con uno de los entrenudos más grande que los demás. Hojas maculadas

Oeceoclades maculata

26b. Pseudobulbos o cormos con los entrenudos de tamaño similar. Hojas no maculadas _...……………..... 27

27a. Cormos hipógeos. Pseudobulbos ausentes. Hojas 2 ................................................. Govenia utriculata vel aff.

27b. Cormos hipógeos ausentes. Pseudobulbos presentes. Hojas 3 ó más ……………………..................... 28

28a. Pseudobulbos de más de $15 \mathrm{~cm}$ de largo. Labelo verrugoso ................................ Cyrtopodium aff. willmorei

28b. Pseudobulbos de menos de $12 \mathrm{~cm}$ de largo. Labelo no verrugoso …............................................. 29

29a. Inflorescencias multifloras, más largas que el pseudobulbo. Flores de color púrpura intenso. Labelo cuando aplanado conspicuamente 3-lobulado

Mormodes atropurpurea

29b. Inflorescencias multifloras, más cortas que el pseudobulbo. Flores amarillas. Labelo cuando aplanado, ligeramente 3-lobulado

Mormodes sp.

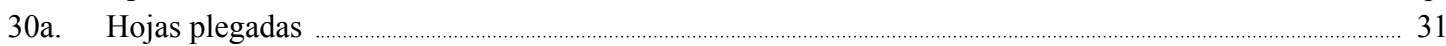

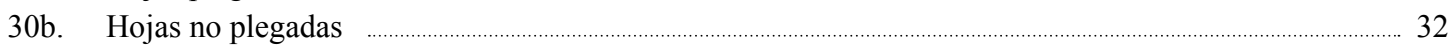

31a. Flores amarillas-marrones, con manchas pardo-rojizas; pétalos linear-lanceolados, de márgenes enteros, basalmente fusionados con la columna; labelo sin una mancha parecida a un ojo a cada lado de su porción basal, mesoquilo no desarrollado

Gongora maculata

31b. Flores amarillas; pétalos aovado-elípticos, márgenes ondeado-rizados, no fusionados con la columna; labelo con una mancha parecida a un ojo a cada lado de su porción basal, mesoquilo desarrollado

Stanhopea wardii

32a. Tallos secundarios sobrepuestos Scaphyglottis fusiformis

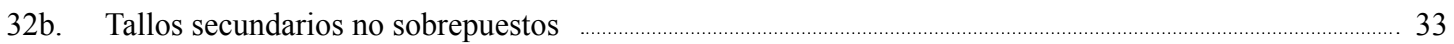

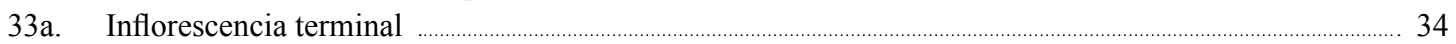

33b. Inflorescencia basal o en las vainas de las hojas jóvenes, pero nunca terminal …...................................... 39

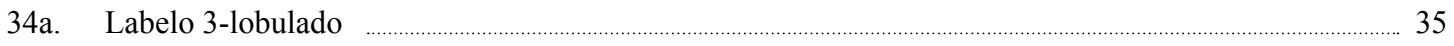

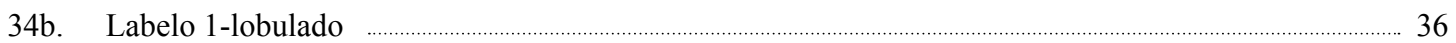

35a. Flores resupinadas, más de $1 \mathrm{~cm}$ de diámetro; labelo con una mancha púrpura en la base del lóbulo medio

Encyclia cordigera

35b. Flores no resupinadas, menos de $0.5 \mathrm{~cm}$ de diámetro; labelo sin una mancha púrpura en la base del lóbulo medio Polystachya foliosa

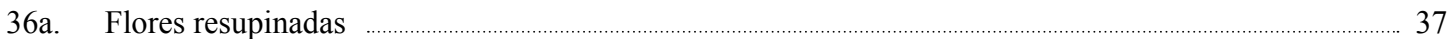

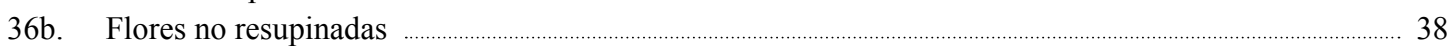

37a. Flores moradas, más de $3 \mathrm{~cm}$ de diámetro; labelo no verrugoso ……......................... Cattleya gaskelliana

37b. Flores blanco-cremoso con detalles marrones, menos de $1 \mathrm{~cm}$ de diámetro; labelo conspicuamente verrugoso

Prosthechea livida

38a. Pseudobulbos 2-foliados. Callo pubescente en la base del labelo …………....... Prosthechea chacaoensis

38b. Pseudobulbos 1-foliados. Callo glabro en la base del labelo ……....................... Prosthechea venezuelana

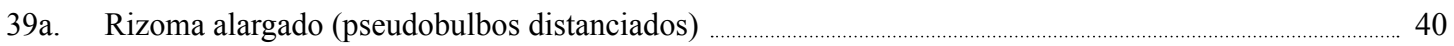

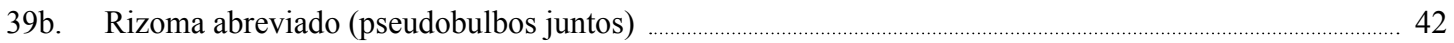

40a. Flores agrupadas en una inflorescencia racemosa. Sépalos laterales fusionados en un sinsépalo conduplicado y geniculado

Rodriguezia lanceolata 
40b. Flores solitarias o en fascículos. Sépalos laterales libres o connados en la base, nunca geniculados ..... 41

41a. Flores solitarias o en grupos de 2; nacen en las vainas de las hojas jóvenes; sépalos laterales libres entre sí; labelo pubescente en su base Camaridium ochroleucum

41b. Flores agrupadas en inflorescencias densamente fasciculadas, basales al pseudobulbo; sépalos laterales connados; labelo glabro

Camaridium micranthum

42a. Flores solitarias

42b. Flores agrupadas en inflorescencias

43a. Plantas no cespitosas, más de $10 \mathrm{~cm}$ de alto. Flores de más de $1 \mathrm{~cm}$ de diámetro; labelo con el callo basalmente glabro Maxillaria porrecta

43b. Plantas cespitosas, menos de $7 \mathrm{~cm}$ de alto. Flores de menos de $0.5 \mathrm{~cm}$ de diámetro; labelo con el callo basalmente pubescente Maxillariella caespitifica

44a. Labelo simple 45

44b. Labelo 3-lobulado 46

45a. Plantas verdes, más de $10 \mathrm{~cm}$ de alto. Pseudobulbos bifoliados. Inflorescencia con más de 5 flores. Flores más de $2 \mathrm{~cm}$ de diámetro; labelo con el ápice acuminado Brassia wageneri

45b. Plantas rojizas, menos de $7 \mathrm{~cm}$ de alto. Pseudobulbos unifoliados. Inflorescencia con 2-4 flores. Flores menos de $1 \mathrm{~cm}$ de diámetro; labelo apicalmente emarginado

46a. Sépalos connados; pétalos con márgenes ondulados Leochilus labiatus

46b. Sépalos libres; pétalos con márgenes enteros Gomesa bicolor Oncidium obryzatum

\section{Descripciones}

Acianthera lanceana (Lodd. ex Lindl.) Pridgeon \& M.W.Chase, Lindleyana 16(4): 244. 2001. Fig. 1B.

Epífita, cespitosa, menos de $12 \mathrm{~cm}$ de alto. Inflorescencia racemosa. Flores variando desde verdes hasta naranja, de menos de $1 \mathrm{~cm}$ de diámetro; sépalos externamente hirsutos o tuberculados, los laterales unidos en un sinsépalo bífido; pétalos lanceolados, con márgenes fimbriados. Ovario glabro. En la zona existe una variedad cleistógama. Florece entre octubre y marzo.

Acianthera miqueliana (H. Focke) Pridgeon \& M.W.Chase, Lindleyana 16: 244. 2001.

Epífita, cespitosa, rizoma rastrero, cubierto por vainas. Ramicaules usualmente morados, menos de $10 \mathrm{~cm}$ de alto. Hojas usualmente de color purpúreo. Flores solitarias, sucesivas; sépalos laterales unidos en un sinsépalo; pétalos lanceolados, de menor tamaño que los sépalos. Florece entre agosto y noviembre. El material aquí referido, a esta especie, pudiese representar el concepto Acianthera angustifolia (Lindl.) Luer.

Acianthera rubroviridis (Lindl.) Pridgeon \& M.W. Chase, Lindleyana 16(4): 246. 2001.
Epífita, más de $15 \mathrm{~cm}$ de alto. Rizoma abreviado. Flores fasciculadas, usualmente en número de dos, verdes hasta amarillentas, de menos de $1 \mathrm{~cm}$ de diámetro; sépalos externamente hirsutos, los laterales connados hasta más o menos la mitad de su longitud; pétalos flabelados, márgenes enteros. Ovario pubescente. Florece entre octubre y abril.

Anathallis sclerophylla (Lindl.) Pridgeon \& M.W. Chase, Lindleyana 16(4): 250. 2001.

Epífita. Ramicaules de menos de $25 \mathrm{~cm}$ de alto. Inflorescencia racemosa, multiflora. Flores de menos de $2 \mathrm{~cm}$ de diámetro, blanquecinas, sépalos pubescentes en la cara interna, largamente acuminados, los laterales connados en la base; pétalos lanceolados, más pequeños que los sépalos. Florece entre julio y agosto.

Brassia wageneri Rchb.f., Bonplandia 2: 14. 1854.

Epífita. Pseudobulbos heteroblásticos, bifoliados, hojas coriáceas. Inflorescencia racemosa, basal al pseudobulbo. Flores de más de $3 \mathrm{~cm}$ de diámetro, verdi-amarillentas con manchas marrones, sépalos y pétalos linear-lanceolados; labelo simple, obovadorómbico, acuminado. Florece entre marzo y mayo. 
Camaridium micranthum M. A. Blanco, Lankesteriana 7(3): 520. 2007. Fig. 1C.

Epífita. Rizoma alargado (pseudobulbos muy separados) y conspicuamente cubierto de vainas. Pseudobulbos heteroblásticos, unifoliados. Inflorescencia densamente fasciculada en la base del pseudobulbo. Flores blancas, de menos de $0.5 \mathrm{~cm}$ de diámetro; sépalos aovado-lanceolados, los laterales connados en la base; pétalos aovado lanceolados; labelo glabro, 3-lobulado, lóbulo medio obtuso. Florece entre octubre y diciembre.

Camaridium ochroleucum Lindl., Bot. Reg. 10: t. 844. 1824.

Epifita. Rizoma alargado (pseudobulbos muy separados) y conspicuamente cubierto de vainas foliares. Pseudobulbos heteroblásticos, uni- o bifoliados. Flores solitarias o en grupos de 2; nacen en las vainas de hojas jóvenes, cerca del ápice del tallo. Flores de más de $1 \mathrm{~cm}$ de diámetro, blancas, labelo amarillo; sépalos y pétalos lanceolados, libres entre sí; labelo 3-lobulado, pubescente o papiloso en su base. Florece entre mayo y julio.

Campylocentrum micranthum (Lindl.) Rolfe, Orchid Rev. 11(128): 245. 1903.

Epífita, crecimiento monopodial. Hojas coriáceas. Inflorescencia racemosa; nacen por debajo de cada raíz. Flores de menos de $0.5 \mathrm{~cm}$ de diámetro, de color blanco hasta cremosas; labelo espolonado. Florece entre noviembre y marzo.

Cattleya gaskelliana Rchb.f., Gard. Chron., n.s. 19: 243. 1883. Fig. 1D--E.

Epífita. Pseudobulbos heteroblásticos, unifoliados. Inflorescencia racemosa de 2--5 flores, de más de $5 \mathrm{~cm}$ de diámetro, lila hasta blancas, fragantes, usualmente con una mancha morada hacia el ápice del labelo; labelo simple, subpandurado, en posición natural abrazando a la columna, margen apical rizado y ligeramente emarginado. Usualmente florece en mayo; sin embargo, es posible encontrar ejemplares en floración entre noviembre-diciembre.

Cyrtopodium aff. willmorei Knowles \& Westc., Fl. Cab. 1: t. 4. 1837.

Terrestre. Pseudobulbos homoblásticos, de más de $20 \mathrm{~cm}$ de alto. Hojas plegadas. Inflorescencia paniculada, basal. Flores anaranjado verdoso, con manchas pardas; labelo 3-lobulado, verrugoso. Florece en mayo.

Dimerandra emarginata (G.Mey.) Hoehne, Bol. Agric. (Sao Paulo) 34: 618. 1934.

Epífita. Rizoma abreviado. Tallos secundarios de menos de $50 \mathrm{~cm}$ de alto, ligeramente engrosados hasta formar cañas, los viejos a veces huecos. Hojas dísticas, dispersas por el tallo. Flores rosadas, solitarias sucesivas, raro 2--3 en antesis simultánea; labelo con callo formado por una serie de lamelas transversales. Florece entre abril y junio.

Elleanthus graminifolius (Barb.Rodr.) Løjtnant, Bot. Not. 129(4): 447-448, 450, f. 2. 1977.

Epífita. Rizoma abreviado. Tallos secundarios de menos de $25 \mathrm{~cm}$ de alto. Hojas lineares, hacia la mitad superior del tallo secundario. Inflorescencia terminal, espiciforme; brácteas conspicuas, persistentes, de color marrón. Flores blancas, diminutas; labelo con el margen apical ciliado, columna pubescente. Florece entre diciembre y enero.

Encyclia cordigera (Kunth) Dressler, Taxon 13(7): 247. 1964.

Epífita. Rizoma abreviado. Pseudobulbos heteroblásticos, bifoliados. Inflorescencia terminal, racimo hasta panícula. Flores de más de $2 \mathrm{~cm}$ de diámetro, con sépalos y pétalos carnosos, de color marrón hasta púrpura; labelo 3-lobulado, blanco con una mancha púrpura en la base del lóbulo medio, lóbulo medio emarginado. Florece entre marzo y mayo.

Epidendrum anceps Jacq., Select. Stirp. Amer. Hist. 224, pl. 138. 1763. Fig. 1F.

Epífita. Rizoma abreviado. Crecimiento simpodial. Hojas coriáceas, distribuidas a lo largo del tallo secundario, usualmente con un color algo purpúreo. Inflorescencia racimo o panícula subcapitada. Flores de color marrón-cremoso, menos de $1 \mathrm{~cm}$ de diámetro; labelo truncado-retuso en su ápice, con márgenes enteros. Florece entre septiembre y diciembre.

Epidendrum ciliare L., Syst. Nat. (ed. 10) 2: 1246. 1759. Fig. 1G.

Epífita. Rizoma abreviado. Pseudobulbos heteroblásticos uni- o bifoliados. Inflorescencia 


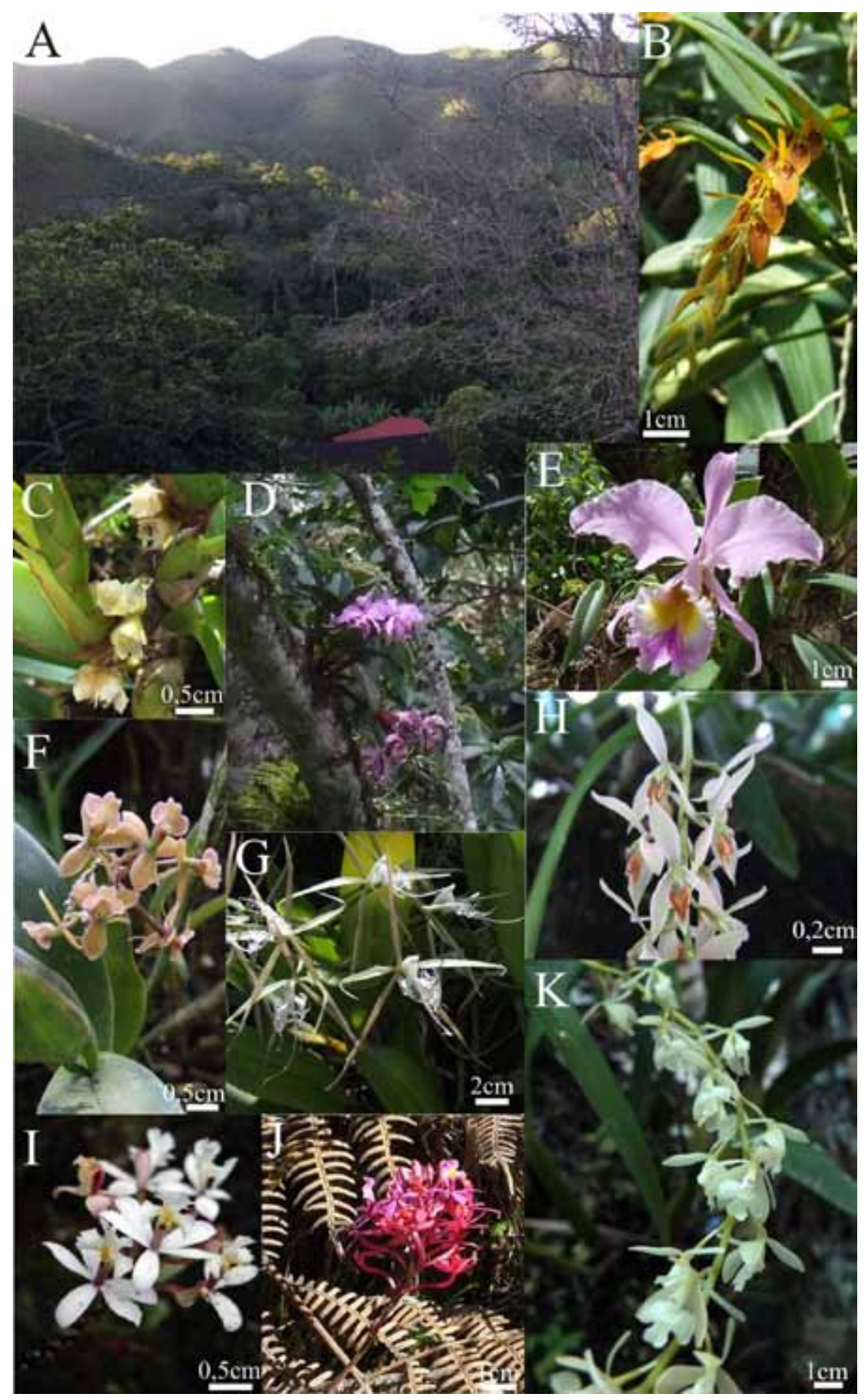

Figura 1. Panorámica de una porción de la Hacienda La Cuchilla y algunas especies encontradas en los cafetales. A vista de una parte de la Hacienda La Cuchilla. B - Acianthera lanceana. C - Camaridium micranthum. D, E Cattleya gaskelliana creciendo silvestre y detalle de flor en cultivo. F - Epidendrum anceps. G - E. ciliare. H - E. ferrugineum. I, J - E. secundum vel sp.aff. en sus dos variedades. K. E. subpurum. 
terminal, racemosa. Flores de más de $4 \mathrm{~cm}$ de diámetro, blancas; labelo 3-lobulado, lóbulo medio acicular, lóbulos laterales con los márgenes externos irregularmente laciniados. Florece entre noviembre y enero.

Epidendrum ferrugineum Ruiz \& Pav., Syst. Veg. Fl. Peruv. Chil. 1: 245. 1798. Fig. 1H.

Epífita. Rizoma abreviado. Tallos secundarios aplanados. Hojas coriáceas. Inflorescencia terminal, racemosa, compacta, péndula, con vainas espatáceas en su base. Flores de menos de $1 \mathrm{~cm}$ de diámetro, de color blanco cremoso; labelo de color naranja con detalles azulados, 3-lobulado, lóbulo medio agudo. Florece entre agosto y noviembre

Epidendrum ramosum Jacq., Enum. Syst. P1. 29. 1760.

Epífita. Hojas coriáceas, lineares. Inflorescencias terminales, fractiflexas, con brácteas conspicuas, infundibuliformes, coriáceas. Flores de menos de 0.5 $\mathrm{cm}$ de diámetro, bronceadas, duras al tacto; labelo simple, agudo. Ovario con una vesícula alargada en su extremo apical. Florece entre octubre y diciembre.

Epidendrum rigidum Jacq., Enum. Syst. P1., 29. 1760.

Epífita. Rizoma muy ramificado. Hojas dísticas, carnoso-coriáceas. Inflorescencias terminales, racemosas, brácteas conspicuas, verdes, coriáceas, infundibuliformes, ocultando el ovario. Flores verdes, de menos de $0.5 \mathrm{~cm}$ de diámetro, perianto rígido al tacto; labelo aovado, obtuso. Ovario sin una vesícula. Florece entre agosto y febrero.

Epidendrum secundum Jacq. vel aff., Enum. Syst. P1., 29. 1760. Fig. 1I--J.

Epífita o terrestre. Crecimiento monopodial. Hojas dísticas, coriáceas o carnoso coriáceas. Inflorescencia terminal, racemosa, con las flores concentradas hacia el ápice. Flores moradas o blancas, de menos de $1 \mathrm{~cm}$ de diámetro; labelo 3-lobulado, márgenes irregularmente dentado-laciniados. Esta planta tiene la capacidad de producir plántulas en el eje floral a partir de yemas que no se desarrollaron en flores. Florece entre noviembre y mayo. En la Serranía de la Cuchilla y otros sectores del Turimiquire aparentemente sólo se ha colectado una entidad que puede presentar morfos blancos ó lila. Sin embargo, algunos investigadores han propuesto que realmente la variabilidad morfológica de esta especie, básicamente en tamaños y colores de las flores, especialmente en localidades de Guayana o la Cordillera de la Costa (sector centro-occidental) hace que amerite una revisión con el fin de definir si está es una entidad muy variable o un complejo de especies.

Epidendrum aff. siphonosepalum Garay \& Dunst., Venez. Orchid. Ill 5: 102. 1972.

Epífita. De menos de $10 \mathrm{~cm}$ de alto. Crecimiento monopodial. Hojas dísticas, glaucas. Por su forma y posición de flores secas y capsulas se presume que la entidad mencionada aquí pertenece a esta especie; sin embargo, no se conoce su flor.

Epidendrum subpurum Rchb.f., Bonplandia 2: 21. 1854. Fig. 1K.

Epífita. Tallos secundarios algo engrosados, pero sin llegar a formar pseudobulbos. Hojas agrupadas hacia el ápice de estos. Inflorescencia terminal, usualmente una panícula laxa. Flores blancas, fragantes en la noche, de menos de $1 \mathrm{~cm}$ de diámetro; sépalos lanceolados, los laterales algo oblicuos; pétalos linearfiliformes; labelo 3-lobulado, márgenes enteros, lóbulo medio emarginado. Florece entre febrero y mayo.

Gomesa bicolor (Lindl.) M.W.Chase \& N.H.Williams, Ann. Bot. (Oxford) 104: 396. 2009. Fig. 2H.

Epífita. Crecimiento simpodial. Pseudobulbos heteroblásticos, bifoliados. Inflorescencia racemosa, basal al pseudobulbo. Flores amarillas, mayores de $1.5 \mathrm{~cm}$ de diámetro; sépalos laterales connados hasta más o menos la mitad de su longitud; pétalos con márgenes ondulados; labelo 3-lobulado, lóbulo medio emarginado. Florece entre octubre y diciembre. Esta entidad conocida también como Oncidium bicolor Lindl., pertenece a un grupo básicamente brasilero de sépalos laterales parcialmente fusionados, labelo con un lóbulo central muy grande, algo elíptico, un callo plurilamelado y una columna con alas conspicuas; se diferencia profundamente de otras entidades asociadas al concepto Gomesa (tipificado por Gomesa recurva R. Br.), en las que las plantas se caracterizan por sépalos laterales parcialmente fusionados, labelo pequeño, carnoso y retrorso, un callo bilamelado y una columna con alas inconspicuas. Sería recomendable una revisión del género Gomesa a fin de generar un sistema compuesto de varios géneros monofiléticos y morfológicamente reconocibles. 
Gongora maculata Lindl., Edwards's Botanical Register 19: t. 1616. 1833. Fig. 2A.

Epífita. Pseudobulbos heteroblásticos, costillados, unifoliados. Hojas plegadas. Inflorescencia basal, racemosa, péndula, pedúnculo de color marrón-rojizo; flores laxamente dispuestas, con el pedicelo curvo, mayores a $2 \mathrm{~cm}$ de diámetro, de color amarillo con machas marrones; pétalos soldados en su base con la columna, algo recorvados en el ápice; labelo complejo. Florece entre marzo-mayo.

Govenia utriculata vel sp. aff. (Sw.) Lindl., Edwards's

Bot. Reg. 25: Misc. 46 [47]. 1839. Fig. 2B.

Terrestre. Cormos homoblásticos. Hojas 2, plegadas, con largos pecíolos cubiertos con 2 vainas, que forman en conjunto un pseudotallo. Inflorescencia axilar, racemosa. Flores agrupadas hacia el ápice, blancas, menos de $2 \mathrm{~cm}$ de diámetro, con diminutas manchas rosado pálido en la cara interior de los pétalos y manchas de color marrón oscuro en el labelo; labelo simple, ligeramente unguiculado, aovado. Florece entre agosto y septiembre. El nombre Govenia utriculata está tipificado por una entidad descrita para las antillas, por lo que es poco probable que el material venezolano sea igual a éste; sin embargo, a falta de estudios sobre las Govenia de Suramérica se ha preferido mantener una posición conservadora y adoptar tentativamente este nombre para la entidad mencionada aquí.

Isochilus linearis (Jacq.) R.Br., Hort. Kew. (ed. 2) 5: 209. 1813. Fig. 2C.

Epífita. Rizoma abreviado. Hojas linearlanceoladas, coriáceas, distribuidas a lo largo del tallo secundario. Flores terminales, solitarias o en grupos de dos, sucesivas, rosadas, de menos de $0.5 \mathrm{~cm}$ de diámetro; sépalos laterales connados entre sí y con el sépalo dorsal en aproximadamente la mitad de su longitud; labelo lanceolado, obtuso. Florece entre noviembre y diciembre.

Jacquiniella globosa (Jacq.) Schltr., Repert. Spec. Nov. Regni Veg. Beih. 7: 124. 1920.

Epífita, menos de $10 \mathrm{~cm}$ de alto. Hojas dísticas, teretes. Flores rosadas, diminutas, solitarias o en fascículos en el ápice de los tallos secundarios.

Jacquiniella teretifolia (Sw.) Britton \& P. Wilson, Sci. Surv. Porto Rico \& Virgin Islands 6(3): 340. 1926.
Epífita. Crecimiento simpodial, usualmente entre $15--30 \mathrm{~cm}$ de alto. Hojas dísticas, teretes. Inflorescencia terminal, con un largo pedicelo, en el que acompañando a las flores hay 1--2 brácteas foliáceas, carácter distintivo de esta planta.

Leochilus labiatus (Sw.) Kuntze, Revis. Gen. P1. 2: 656. 1891. Fig. 2D.

Epífita. Pseudobulbos heteroblásticos, unifoliados. Inflorescencias racemosas, basales al pseudobulbo, usualmente con 2--3 flores en antésis. Flores de menos de $0.5 \mathrm{~cm}$ de diámetro, sépalos y pétalos de color marrón-rojizo, lanceolados; labelo simple, emarginado, de color amarillo-verdoso con puntos pardos. Un detalle peculiar de esta planta es que es de color rojizo. Florece entre mayo y agosto.

\section{Lockhartia sp.}

Epífita, crecimiento simpodial, de más de $20 \mathrm{~cm}$ de alto. Tallos péndulos. Hojas imbricadas, lateralmente aplanadas. Flores desconocidas.

Maxillaria porrecta Lindl., Edwards's Bot. Reg. 24: misc. 92. 1838. Fig. 2E.

Epífita, rizoma abreviado. Pseudobulbos heteroblásticos, unifoliados. Flores solitarias, basales al pseudobulbo, de más de $2 \mathrm{~cm}$ de diámetro, naranja; labelo 3-lobulado, callo glabro. Florece entre diciembre $\mathrm{y}$ enero.

Maxillariella caespitifica (Rchb.f.) M. A. Blanco \& Carnevali, Lankesteriana 7(3): 528. 2007. Fig. 2F.

Epífita, cespitosa, de menos de $10 \mathrm{~cm}$ de alto. Flores solitarias, basales al pseudobulbo, de color naranja-verdoso, con el labelo con detalles púrpura oscuro; labelo 3-lobulado, pubescente en la base del callo. Florece entre marzo y junio.

Mormodes atropurpurea Lindl., Nat. Syst. Bot. 2: 446. 1836.

Epífita o terrestre. Rizoma abreviado. Pseudobulbos homoblásticos, plurifoliados. Hojas plegadas, caducas, lanceoladas, acuminadas. Inflorescencia racemosa, compacta; nace en la porción media del pseudobulbo, usualmente cuando éste no tiene hojas. Flores púrpura; labelo con una lámina fuertemente reflexoconduplicada, 3-lobulada. Florece entre diciembre y marzo. 


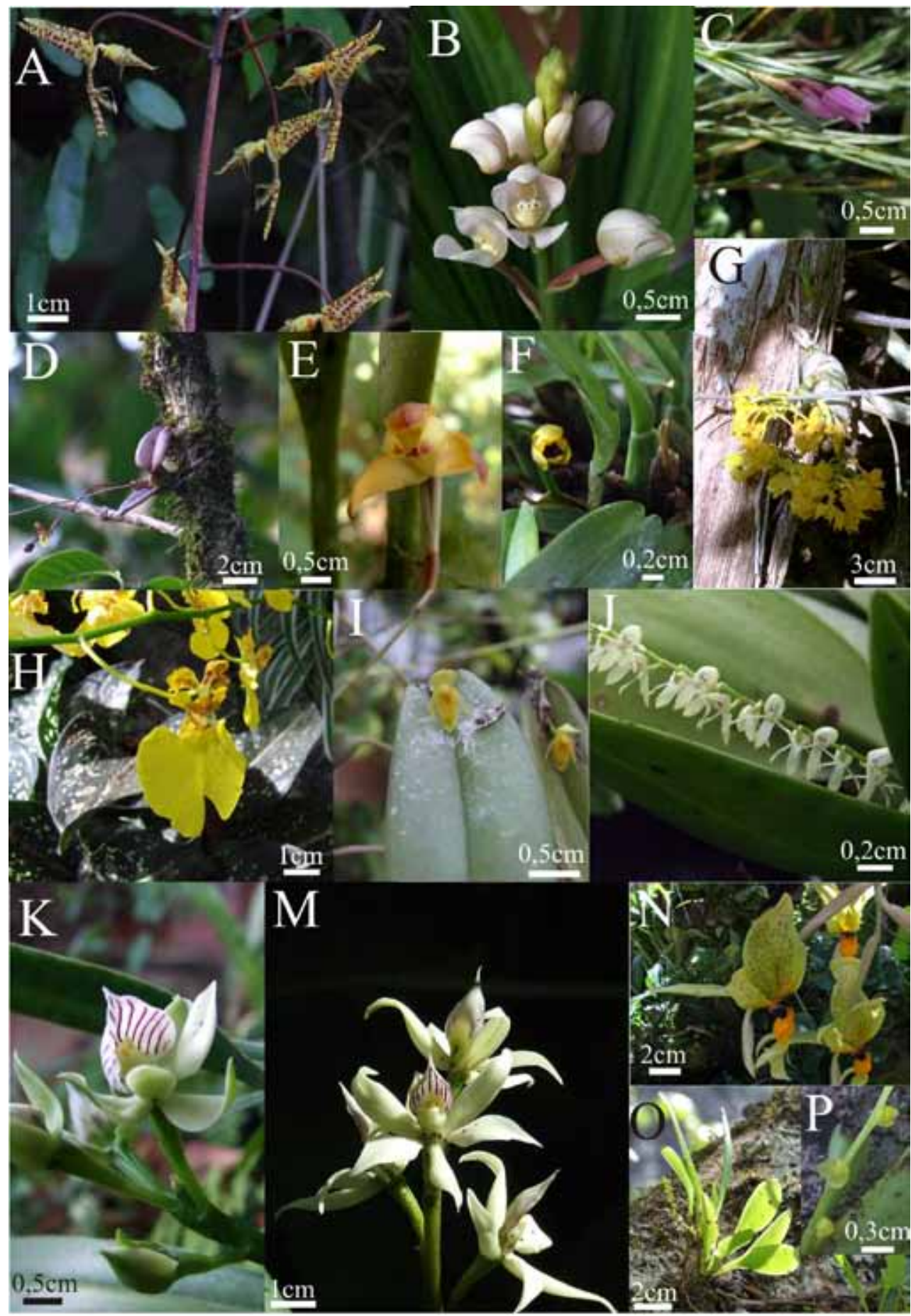

FIgURA 2. Algunas especies encontradas en la Hacienda La Cuchilla. A — Gongora maculata Lindl.. B — Govenia utriculata vel aff.. C - Isochilus linearis. D - Leochilus labiatus. E - Maxillaria porrecta. F - Maxillariella caespitifica. G - Mormodes sp. H - Gomesa bicolor. I — Pleurothallis discoidea. J — P. revoluta. K — Prosthechea chacaoensis. $\mathrm{M}$ - P. venezuelana. $\mathrm{N}$ - Stanhopea wardii. O, P — Stelis cucullata y detalle de su flor. 
Mormodes sp. Fig. 2G.

Epífita o terrestre. Rizoma abreviado. Pseudobulbos homoblásticos, plurifoliados. Hojas plegadas, caducas, lanceoladas, acuminadas. Inflorescencia racemosa, compacta; nace en la porción media del pseudobulbo, usualmente cuando éste no tiene hojas. Flores amarillas, labelo con una lámina fuertemente reflexoconduplicada, ligeramente 3-lobulada. Florece entre diciembre y marzo.

Oeceoclades maculata (Lindl.) Lindl., Gen. Sp.

Orchid. P1. 237-238. 1833.

Terrestre. Crecimiento simpodial. Pseudobulbos heteroblásticos, unifoliados. Hojas maculadas. Inflorescencia basal al pseudobulbo, racemosa. Flores blancas, labelo blanco con rayas rosadas; labelo 3-lobulado, espolonado. Florece entre junio y noviembre, aunque si las condiciones de humedad y temperatura están dadas puede florecer en cualquier momento del año.

Oncidium obryzatum Rchb.f., Bonplandia 2(9): 108109. 1854.

Epífita. Pseudobulbos heteroblásticos, unifoliados. Inflorescencia racemosa o con más frecuencia una panícula, basal al pseudobulbo. Flores amarillas, menores de $1.5 \mathrm{~cm}$ de diámetro; sépalos laterales libres entre sí; pétalos con márgenes enteros; labelo 3-lobulado, lóbulo medio profundamente emarginado. Florece entre octubre y diciembre.

Pleurothallis discoidea Lindl., Edwards's Bot. Reg.

21: sub t. 1797. 1835. Fig. 2I.

Epífita, cespitosa. Ramicaules de menos de 10 $\mathrm{cm}$ de alto. Hojas a veces de color purpúreo. Flores solitarias o raro 2, sucesivas, de color amarillo claro, algo hialinas, menores a $0.5 \mathrm{~cm}$ de diámetro; sépalos laterales formando un sinsépalo; pétalos lineares, de longitud similar a la de los sépalos. Florece entre noviembre y enero; sin embargo, en condiciones de cultivo presenta flores casi todo el año.

Pleurothallis pruinosa Lindl., Edwards's Bot. Reg. 28: Misc. 75-76. 1842.

Epífita, cespitosa. Ramicaules menores a $7 \mathrm{~cm}$ de alto. Inflorescencia racemosa, con 2--5 flores laxamente distribuidas por el eje de la inflorescencia. Flores de menos de $0.3 \mathrm{~cm}$ de diámetro, cremosas; sépalos laterales unidos en un sinsépalo obtuso; pétalos lineares, de longitud similar a los sépalos. Florece entre noviembre y enero.

Pleurothallis revoluta (Ruiz \& Pav.) Garay, Caldasia 8(40): 520. 1962. Fig. 2J.

Epífita. Ramicaules de menos de $25 \mathrm{~cm}$ de alto. Inflorescencias racemosa, multiflora. Flores de menos de $0.5 \mathrm{~cm}$ de diámetro, cremosas; sépalos laterales unidos en un sinsépalo obtuso; pétalos linearfiliformes, de longitud similar a la de los sépalos; labelo geniculado. Florece entre junio y noviembre.

Polystachya foliosa (Hook.) Rchb.f., Ann. Bot. Syst., 6(4): 640. 1863.

Epífita, de hasta $30 \mathrm{~cm}$ de alto. Pseudobulbos homoblásticos, usualmente ocultos por vainas foliares. Inflorescencia terminal, racemosa o más frecuentemente paniculada. Flores no resupinadas, verdes, de menos de $0.5 \mathrm{~cm}$ de diámetro; labelo 3-lobulado, con un callo elevado y cónico en su base. Florece entre noviembre y diciembre.

Prosthechea chacaoensis (Rchb.f.) W.E. Higgins, Phytologia 82(5): 377.1997 [1998]. Fig. 2K.

Epífita, hasta $35 \mathrm{~cm}$ de alto. Pseudobulbos heteroblásticos, bifoliados. Inflorescencia terminal al pseudobulbo, racemosa. Flores no resupinadas, cremosas, con líneas de color púrpura en el labelo; labelo cortamente acuminado, con un callo subcuadrado y pubescente en su base. Las plantas en condiciones de campo y de cultivo suelen diferir sustancialmente en el diámetro de sus flores (en hasta $0.5 \mathrm{~cm}$ ). Florece entre mayo y junio.

Prosthechea livida (Lindl.) W.E. Higgins, Phytologia 82(5): 379. 1997[1998].

Epífita, hasta $25 \mathrm{~cm}$ de alto. Pseudobulbos heteroblásticos, bifoliados. Inflorescencia terminal al pseudobulbo, racemosa. Flores resupinadas, de color blanco cremoso con detalles marrones; labelo conspicuamente verrugoso; columna con una proyección conspicua en su ápice. Florece entre marzo y mayo.

Prosthechea venezuelana (Schltr.) W.E. Higgins, Phytologia 82(5): 381. 1997[1998]. Fig. 2M.

Epífita, hasta $35 \mathrm{~cm}$ de alto. Pseudobulbos 
heteroblásticos, fusiformes, unifoliados. Inflorescencia terminal al pseudobulbo, racemosa. Flores no resupinadas, blancas, labelo blanco con líneas púrpura, acuminado, basalmente con un callo subcuadrado, glabro. Florece entre febrero y mayo.

Rodriguezia lanceolata Ruiz \& Pav., Syst. Veg. Fl. Peruv. Chil., 1: 219. 1798.

Epífita. Rizoma algo alargado. Pseudobulbos heteroblásticos, unifoliados. Inflorescencia basal al pseudobulbo, usualmente algo arqueadas. Flores rosadas hasta rojizas; sépalos laterales unidos en un sinsépalo conduplicado y geniculado; labelo con márgenes algo ondeados, emarginado. Florece entre marzo y mayo.

Scaphyglottis fusiformis (Griseb.) R.E.Schult., Bot. Mus. Leafl. 17(7): 205. 1956.

Epífita. Rizoma abreviado, crecimiento simpodial. Pseudobulbos unifoliados, prolíficos (crecen sobrepuestos), unifoliados. Flores solitarias, blancoverdosas, menores a $0.5 \mathrm{~cm}$ de diámetro; labelo simple, apiculado. Florece entre marzo y mayo.

Stanhopea wardii Lodd. ex Lindl., Sert. Orchid. t. 20. 1836. Fig. $2 \mathrm{~N}$.

Epífita. Rizoma abreviado. Crecimiento simpodial. Pseudobulbos heteroblásticos, costillados, unifoliados. Hojas plegadas. Inflorescencia racemosa, basal, colgante. Flores amarillas con manchas marrones; pétalos con márgenes ondeado-rizados, libres de la columna; labelo complejo (con hipóquilo, mesóquilo y epíquilo), cerca de su base con una mancha casi negra. Florece entre agosto y septiembre.

Stelis cucullata Ames, Sched. Orch. 6: 52-53. 1923. Fig. 2O--P.

Epífita, cespitosa. Ramicaules de menos de 15 $\mathrm{cm}$ de alto. Inflorescencia racemosa, erecta. Flores blanquecinas; sépalos connados en la base, dando a la flor el contorno de un triangulo; pétalos flabelados; labelo obtuso. Florece entre abril y junio.

Stelis pygmaea Cogn., Symb. Antill. 6: 390. 1910.

Epífita, cespitosa, crecimiento simpodial. Ramicaules de menos de $10 \mathrm{~cm}$ de alto. Inflorescencia racemosa, laxas con 2--4 flores. Flores blancoverdosas; sépalos unidos en más de la mitad de su longitud, dando a la flor el contorno de un triángulo; labelo apiculado. Florece entre marzo y mayo.

Agradecimientos. A Günter Gerlach por la identificación de Gongora maculata Lindl., a Luis J. Cumana, Hernán Ferrer, Ivelise de Franco, Germán Carnevali y a los revisores anónimos por la lectura y críticas hechas a las primeras versiones del manuscrito, a José Imery por la revisión del Abstract y a Café San Agustín Dos C.A. por el financiamiento parcial de este proyecto.

\section{LiTERATURA CITADA}

Carnevali G., I. Ramírez-Morillo, G. Romero-González, C. Vargas, \& E. Foldats. 2003. Orchidaceae. Pp. 200-618 in:

Steyermark, J. A., P.E. Berry \& B.K. Holst (Gen. eds.). P.E. Berry, B.K. Holst \& K. Yatskievych (vol. eds.). Fl. Venez. Guayana. Volume 7: Myrtaceae-Plumbaginaceae. Missouri Botanical Garden, St. Louis; Timber Press, Portland.

Carnevali G., G.A. Romero, E. Noguera. \& G. Gerlach. 2007. La familia Orchidaceae en Venezuela: diversidad y biogeografía. Memorias XVII Congreso Venezolano de Botánica: 21-23.

Chase M., J. Freudenstein, K. Cameron \& R. Barrett. 2003. DNA data and Orchidaceae systematics: a new phylogenetic classification. Pp. 69-89 in: Dixon, K., S. Kell, R. Barrett y P. Cribb (eds.). Orchid conservation. Natural History Publications. Kota Kinabalu.

Dressler R. 1981. The Orchids: Natural History and Classification. Harvard University Press. Cambridge, Massachusetts, Estados Unidos.

Dunsterville G. \& L. Garay. 1959. Venezuelan Orchids Illustrated. Vol. 1. André Deutsch Limited, London. Dunsterville G. \& L. Garay. 1961. Venezuelan Orchids Illustrated. Vol. 2. André Deutsch Limited, london. Dunsterville G. \& L. Garay. 1965. Venezuelan Orchids Illustrated. Vol. 3. André Deutsch Limited, london. Dunsterville G. \& L. Garay. 1966. Venezuelan Orchids Illustrated. Vol. 4. André Deutsch Limited, London. Dunsterville G. \& L. Garay. 1972. Venezuelan Orchids Illustrated. Vol. 5. André Deutsch Limited, London. Dunsterville G. \& L. Garay. 1976. Venezuelan Orchids Illustrated. Vol. 6. André Deutsch Limited, London. Figueroa, M. 1992. Flora (Angiospermopsida) del Parque Nacional "El Guácharo" Sector "Cerro La Cueva". Tesis de Maestría. Universidad de Oriente. Jusepín, Venezuela. 
Foldats E. 1969. Orchideaceae. En: Lasser T. (ed.). Flora de Venezuela. Tomo XV. I. Parte. Editorial Instituto Botánico. Caracas, Venezuela.

Foldats E. 1970a. Orchideaceae. En: Lasser T. (ed.). Flora de Venezuela. Tomo XV. II Parte. Editorial Instituto Botánico. Caracas, Venezuela.

Foldats E. 1970b. Orchideaceae. En: Lasser T. (ed.). Flora de Venezuela. Tomo XV. III Parte. Editorial Instituto Botánico. Caracas, Venezuela.

Foldats E. 1970c. Orchideaceae. En: Lasser T. (ed.). Flora de Venezuela. Tomo XV. IV Parte. Editorial Instituto Botánico. Caracas, Venezuela.

Foldats E. 1970d. Orchideaceae. En: Lasser T. (ed.). Flora de Venezuela. Tomo XV. V Parte. Editorial Instituto Botánico. Caracas, Venezuela.

Gentry A. \& C. Dodson. 1987. Diversity and biogeography of neotropical vascular epiphytes. Ann. Missouri Bot. Gard., 74: $205-233$.

Lárez A. 2003. Angiospermas del Parque Nacional El Guácharo, estados Monagas y Sucre. Ernstia 13: 1-28.

Lárez A. 2005. Estado actual del conocimiento de la Flora del Estado Monagas, Venezuela. Revista UDO Agrícola 5: 1-9.

Lindorf H., L. Parisca \& P. Rodríguez. 1999. Botánica, clasificación, estructura y reproducción. Ediciones de la Biblioteca de la Universidad Central de Venezuela. Caracas, Venezuela.

Llamozas S., R. Duno de Stefano, W. Meier, R. Riina, F. Stauffer, G. Aymard O. Huber, \& R. Ortiz. 2003. Libro rojo de la flora venezolana. PROVITA - Fundación POLAR - Fundación Instituto Botánico de Venezuela "Dr. Tobías Lasser". Caracas.

Luer, C. 1986a. Icones Pleurothallidinarum I: Systematics of the Pleurothallidinae. Monogr. Syst. Bot. Missouri Bot. Gard.15: 1-81.

Luer, C. 1986b. Icones Pleurothallidinarum III: Systematics of Pleurothallis. Monogr. Syst. Bot. Missouri Bot. Gard. 20:1109.

Pridgeon A, P. Cribb, M. Chase y F. Rasmussen (eds.). 2003. Genera Orchidacearum, Orchidoideae (part 2): Vanillioideae. Vol. 3. Oxford University Press. Oxford.

Pridgeon A, P. Cribb, M. Chase y F. Rasmussen (eds.). 2005. Genera Orchidacearum, Epidendroideae (part 1). Vol. 4. Oxford University Press. Oxford.

Pridgeon A, P. Cribb, M. Chase y F. Rasmussen (eds.). 2009. Genera Orchidacearum, Epidendroideae (part 2). Vol. 5. Oxford University Press. Oxford.

Schweinfurth, C. 1957. Orchidaceae. En: J. Steyermark (ed.). Contributions to the Flora the Venezuela. Fieldiana, Bot. 28: $845-871$.

Steyermark J. 1966. El Cerro Turimiquire y la región oriental adyacente. Acta Bot. Venez. 1: 104-168.

Steyermark, J. y O. Huber. 1978. Flora del Ávila. Sociedad Venezolana de Ciencias Naturales, Vollmer Foundation y Ministerio del Ambiente y de los Recursos Naturales Renovables. Caracas. 
